This is an Accepted Manuscript of an article published by Taylor \& Francis Group in Psychology, Crime \& Law on 21/02/2017, available online: http://www.tandfonline.com/10.1080/1068316X.2017.1298761 


\section{Psychology, Crime \& Law}

\section{Adapting measures of social climate for use with individuals with intellectual developmental disability in forensic settings}

\section{Natalie Bell, Matthew Tonkin, Verity Chester \& Leam Craig}

To cite this article: Natalie Bell, Matthew Tonkin, Verity Chester \& Leam Craig (2017): Adapting measures of social climate for use with individuals with intellectual developmental disability in forensic settings, Psychology, Crime \& Law, DOI: 10.1080/1068316X.2017.1298761

To link to this article: http://dx.doi.org/10.1080/1068316X.2017.1298761

Accepted author version posted online: 21

Feb 2017.

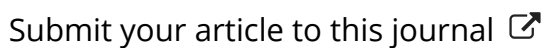

View related articles $₫$

View Crossmark data $₫$ 
Publisher: Taylor \& Francis \& Informa UK Limited, trading as Taylor \& Francis Group Journal: Psychology, Crime \& Law

DOI: $10.1080 / 1068316 X .2017 .1298761$

\section{Adapting measures of social climate for use with individuals with intellectual developmental disability in forensic settings}

Natalie Bell ${ }^{1}$, Matthew Tonkin ${ }^{2}$, Verity Chester $^{3}$ \& Leam Craig ${ }^{1,4}$

${ }^{1}$ Department of Psychology, Birmingham City University, Birmingham, UK

${ }^{2}$ Department of Criminology, University of Leicester, Leicester, UK

${ }^{3}$ Partnerships in Care Learning Disability Services, Diss, UK and University of East Anglia, Norwich, UK

${ }^{4}$ Forensic Psychology Practice Ltd, UK and Centre for Forensic and Criminological Psychology, University of Birmingham, UK

Corresponding Author: Natalie Bell, Birmingham City University, The Curzon Building, 4 Cardigan Street, Birmingham, B4 7BD, UK.

Email: Natalie.Bell@mail.bcu.ac.uk 


\title{
Adapting measures of social climate for use with individuals with intellectual developmental disability in forensic settings
}

\begin{abstract}
The social climate of forensic settings is thought to impact on a number of important clinical and organisational outcomes and is, therefore, an important construct in relation to the successful functioning of forensic units. A variety of self-report questionnaires have been developed to objectively measure the social climate of forensic settings (e.g. the Correctional Institutions Environment Scale and the Essen Climate Evaluation Schema), however these questionnaires have not been validated for individuals with intellectual developmental disabilities (IDD). Given the prevalence of IDD in prison and forensic psychiatric settings and the potential impact of such cognitive deficits on the ability to complete a range of self-report questionnaires, it is important to consider the potential reliability and validity of existing social climate measures in IDD populations. This article will, therefore: 1 ) examine the cognitive, linguistic and response format difficulties that may arise when administering self-report measures of social climate in IDD populations; 2) consider potential adaptations to existing measures of social climate that might make them more suitable for use with IDD populations; and 3) identify important directions for future research in the area.
\end{abstract}


Keywords: social climate, intellectual disability, developmental disability, forensic psychiatric hospital, prison

\section{Introduction}

Social climate is a crucial aspect of the treatment environment in forensic settings and has been referred to in various ways such as institutional milieu, psychosocial atmosphere, therapeutic milieu, treatment environment and ward atmosphere. The importance of social climate is underscored by research that has shown climate to relate to many important clinical and organisational outcomes including resident and staff satisfaction (Bressington, Stewart, Beer, \& MacInnes, 2011; Røssberg \& Friis, 2004), institutional violence (Friis \& Helldin, 1994), staff morale and stress (Moos \& Schaefer, 1987; Rose, 1993) and treatment engagement and outcomes (Beech \& Hamilton-Giachritsis, 2005; Long et al., 2011; Moos, Shelton, \& Petty, 1973). These relationships underscore the influence of a positive social climate on successful offender rehabilitation, highlighting the necessity of regularly monitoring social climate using a reliable and valid measurement tool.

A variety of self-report questionnaire measures have been developed that might assist the regular monitoring of social climate (see Tonkin, 2015, for a review). Many of these questionnaires have, however, limited evidence to support their reliability and validity with certain populations. For example, none of these questionnaires have been validated for use with people with intellectual developmental disability (IDD) who are in forensic settings. This is a significant limitation given the high prevalence of IDD in forensic settings ${ }^{1}$ and the recent recommendation that social climate be regularly monitored in all UK inpatient IDD

\footnotetext{
${ }^{1}$ The latest Learning Disability Census revealed that there were 3,000 individuals with IDD in specialist inpatient units in England (Health and Social Care Information Centre, 2015). The number of individuals with IDD in prison remains unknown. However, Rack (2005) estimated that $20 \%$ of the prison population has a hidden disability and Mottram (2007) estimated that 32\% of the prison population had an IQ<80.
} 
services (Royal College of Psychiatrists, 2013). It is important that research considers whether existing measures of social climate are suitable for use with IDD populations. Without such consideration, attempts to monitor the social climate and patient experience in IDD settings will be, at best, meaningless and, at worst, misleading.

Thus far, limited consideration has been given to this issue within the academic literature and the current review seeks to fill this gap. Existing questionnaire-based measures of forensic social climate will be discussed, as will the cognitive, linguistic and response format difficulties that may arise when administering these questionnaires with IDD populations. The article will then consider potential adaptations to these measures that might make them more suitable for use with IDD populations, and will finish by identifying important areas for future research.

\section{Questionnaire-based measures of social climate}

A range of questionnaire-based measures exist to assess social climate (and related constructs), but the most widely used measures are the Ward Atmosphere Scale (WAS; Moos, 1974), the Correctional Institutions Environment Scale (CIES; Moos, 1987) and the Essen Climate Evaluation Schema (EssenCES; Schalast et al., 2008). Alternative - less commonly used - questionnaires include the Prison Group Climate Instrument (PGCI; Van der Helm, Stams, \& van der Laan, 2011) and the Prison Preference Inventory (PPI; Toch, 1977).

The social climate of psychiatric settings was first discussed in the literature in the 1960s by Rudolf Moos (e.g. Moos \& Houts, 1968). In the late 1960s and 1970s Moos developed the WAS, the first measure of social climate for psychiatric settings, and subsequently the CIES, an adapted version of the WAS designed to measure the social climate in correctional/forensic settings. The CIES is a self-report questionnaire measure that 
can be completed by both staff and residents of prison and secure hospital units. It incorporates nine of the ten original subscales of the WAS; involvement, support, expressiveness, autonomy, practical orientation, personal problem orientation, order and organisation, clarity and staff control. These nine subscales are combined into three higher order factors; relationships, personal growth and system maintenance. The relationships factor measures how involved residents are on the unit, whether residents support each other and are supported by staff, and whether the programme encourages open expression of residents' feelings. The personal growth factor measures whether residents are encouraged to be independent and to take responsibility for their own decisions, whether units promote practical preparation for residents' release, and whether units help increase residents' levels of self-understanding. The system maintenance factor measures whether the units' function in an organised and coherent way (Moos, 1987). The WAS and CIES were initially the most frequently used measures of social climate with both measures used extensively in forensic settings. However, these scales have been criticised for a variety of reasons, including the outdated content of some items, the length of the questionnaires (100 items in the WAS and 90 items in the CIES), and a lack of evidence to support the scales' proposed factor structures and psychometric properties (Røssberg \& Friis, 2004; Wright \& Boudouris, 1982).

Shortly before Moos adapted the WAS to develop the CIES, the Prison Environment Inventory (PEI; Wright, 1985) was developed. This was based on Toch’s (1977) PPI and, whilst still using the factors developed by Toch and retaining the theoretical basis behind these factors, the adaptations led to a measure with improved reliability and validity (Bradford, 2006; Wright, 1985). The PEI is a 48-item questionnaire that measures social climate across eight factors: privacy, safety, structure, support, emotional feedback, social stimulation, activity and freedom. Few studies have, however, utilised the PEI and, therefore, there is little empirical support for this measure to date. 
The EssenCES was developed in an attempt to overcome some of the limitations of the WAS and CIES and consists of two unscored and 15 scored items across three factors measuring therapeutic hold, patient cohesion and experienced safety. Therapeutic hold refers to the need for a minimum level of hold and support in any therapeutic setting. Patient cohesion indicates whether characteristics of a therapeutic community are present on a unit. Experienced Safety pertains to the fact that safety is a basic human need and that effective treatment cannot take place in an atmosphere of constant threat of violence (Schalast et al., 2008). Originally developed in German and later translated to English, the EssenCES is currently the most widely used measure of social climate in UK forensic settings. The English version of the EssenCES has received considerable empirical support in the United Kingdom and Australia with studies supporting the proposed factor structure and psychometric properties (e.g. Howells et al., 2009; Tonkin et al., 2012). Construct validity has been demonstrated with significant relationships identified between the EssenCES and a number of important clinical and organisational outcomes (e.g. Day, Casey, Vess, \& Huisy, 2012; Tonkin et al., 2012) and the questionnaire has demonstrated the ability to differentiate between different security levels and perceptions of residents and staff (e.g. Day et al., 2012; Long et al., 2011; Milsom, Freestone, Duller, Bouman, \& Taylor, 2014). Despite substantial support of the EssenCES, it is important to note that studies using the EssenCES have not yet sampled the full range of forensic populations. There has, for example, been limited attention paid to low secure settings, women's services and adolescent, IDD and neurobehavioural rehabilitation populations. Consequently, concerns have been raised regarding generalisation of the above results to these populations (Alderman \& Groucott, 2012; Tonkin et al., 2015).

The PGCI was developed as a way of measuring group climate in forensic settings. The authors noted that group climate is a slightly different construct to social climate and, therefore, this measure of climate differs from other existing measures as all items are 
relevant to the context of residents living in groups. The concept of living in groups is more prevalent in adolescent prisons and secure residential hospitals, in comparison to adult prisons where inmates often spend a lot of time in their cells (van der Helm et al., 2011). The PGCI, therefore, incorporates four first-order factors of repression, support, growth and group atmosphere, which are reported to be the four components responsible for the quality of forensic group climate. The PGCI consists of 36 items that were adapted from other measures of prison climate, with these items rated on a five-point Likert scale. Although the PGCI has not been used frequently within social climate research, studies undertaken in Germany and the Netherlands have generally supported the proposed factor structure and have reported satisfactory internal consistency and validity (van der Helm et al., 2011; Heynen, 2016), along with providing preliminary evidence regarding the association between group climate as measured by the PGCI and other variables (e.g. empathy) (van de Helm, Stams, van Genabeek, \& van der Laan, 2012). Although of note is that the majority of studies have been undertaken with adolescent populations, thus the validity of the PGCI with adult populations is less well established.

\section{Measurement of the social climate in ID Settings}

Although knowledge and understanding of the social climate construct have developed considerably since Moos' early research in the 1960s, little attention has been paid to the social climate of specialist forensic populations, including IDD services (Tonkin, 2015).

Social climate literature for this specialist population is therefore sparse. No empirical studies have considered how the social climate of IDD services is constructed, nor are there studies examining the relationships between social climate and outcome variables, such as resident and staff satisfaction, institutional violence, staff morale and stress, or treatment engagement and outcomes in IDD services. 
A small number of studies have examined the social climate of forensic IDD settings using the WAS or CIES. McGee and Woods (1978) used a modified version of the WAS to measure the social climate in a residential adolescent IDD service, with findings highlighting significant differences between staff and resident perceptions of social climate ${ }^{2}$. The authors clearly appreciated the necessity of adapting the WAS for IDD populations, however of concern is that these adaptations may have rendered the scale unreliable and may therefore impact upon the credibility of their findings. Langdon, Swift, and Budd (2006) used the CIES Short Form (Moos, 1987b), which is comprised of the first 36 items of the standard CIES and includes four items from each of the nine subscales, to compare the social climate of medium and low secure forensic IDD units, and staff and resident perceptions of social climate. Their findings indicated that residents across both medium and low secure units rated the units significantly higher than staff on the involvement, support, personal problem orientation and staff control subscales, however they rated the units significantly lower than staff on the practical orientation subscale. Additionally, the low secure unit was rated significantly higher on the practical orientation and personal problem orientation subscales by both staff and residents. The authors concluded that their findings provided some support for the use of the CIES Short Form in IDD populations, however remarked that a lack of normative data for IDD settings made it difficult to contextualise their results.

Whilst it is positive that some research has utilised the WAS and CIES within IDD populations, of note is that, although McGee and Woods (1978) utilised the WAS to conduct their study, the WAS is intended for use in general psychiatric units rather than forensic units. It is also important to highlight that the study conducted by Langdon et al. (2006) used the CIES Short Form. To the authors knowledge there have not been any validation studies conducted using this version of the CIES and, although Moos (1975) reported on

\footnotetext{
${ }^{2}$ Of note is that the authors did not specify how they adapted the WAS for this study.
} 
development of the CIES Short Form using a large sample of juvenile residents and staff, the only reported findings indicated that the CIES Short Form provided profiles similar to those obtained from the standard version of the CIES (interclass correlations between the nine subscales of the standard version of the CIES and the CIES Short Form greater than 0.8 on the majority of units for both resident and staff samples). It is therefore unclear how utilisation of both the WAS and the CIES Short Form may have impacted on the findings of the studies and consequently how much credence should be given to the results. It is also important to highlight that the study conducted by Langdon et al. (2006) only included male residents and that McGee and Woods (1978) did not specify the participating residents’ genders. Furthermore, both studies included small resident samples (10 and 18 residents respectively) within individual forensic establishments. This raises questions as to whether the results of these studies would be generalisable to the broader forensic IDD population.

A small number of studies report on use of the EssenCES in forensic IDD populations. In 2012 Quinn, Thomas, and Chester explored the psychometric properties of the EssenCES in a sample of 37 male and 14 female residents (mean Intelligence Quotient [IQ] 63.21) in a UK medium and low secure IDD service. Their findings indicated acceptable reliability. Construct validity was examined through comparison of resident scores on the medium and low secure units and, although residents on the low secure unit reported significantly higher levels of experienced safety, no significant differences were found on the therapeutic hold or patient cohesion subscales. The authors concluded that it is unclear whether the EssenCES is a valid measure in this population. Willets, Mooney, and Blagden (2014) used the EssenCES to examine social climate across 64 male residents and 73 staff in one establishments' IDD and non-IDD medium and low secure services. One aspect of their study compared the scores of residents with IDD across medium and low secure units and, similar to the findings of Quinn et al., they reported that although there was a statistically 
significant difference on the experienced safety subscale, no differences reached significance on the remaining subscales. These findings lead to the question of whether the patient cohesion and therapeutic hold subscales are measuring the constructs they intend to measure in IDD populations as, in line with previous research using the EssenCES in non-IDD populations, it would be reasonable to expect that units of a higher level of security would be rated as less cohesive and less supportive than units of a lower level of security (Howells et al, 2009; Schalast et al. 2008; Tonkin et al., 2012). However, as it is currently unclear whether the same findings should be expected in IDD settings as non-IDD settings, it is difficult to draw any concrete conclusions with regards to the reliability or validity of the aforementioned results.

Of note is that the aforementioned studies included mainly male participants within medium and low secure units in individual establishments. This results in difficulties generalising the above findings to broader IDD populations. It is also important to recognise that none of the above studies commented on the method of administering the measure or on any difficulties experienced by participants during completion. Furthermore, most studies did not comment on the participants' IQ range and whether any participants were excluded due to cognitive difficulties. Clearly difficulties completing questionnaire measures are more apparent in individuals with more moderate IDD, and it is therefore imperative to identify the IQ range of participants and highlight whether or not all individuals on the units were included in the studies.

The suitability of the EssenCES for IDD populations has been questioned in a recent study which investigated clinician experiences of using the EssenCES in forensic IDD settings (Chester et al., 2015). Participants were seven clinicians currently working, or who had previously worked, within the Psychology discipline in one UK forensic IDD service. The results raised a number of concerns across all items of the scale. Concerns included 
residents' difficulties understanding the language used in the measure (e.g. complex words such as 'progress' and abstract concepts such as 'atmosphere') and difficulties when residents were required to comment on the views and experiences of other residents and staff (e.g. 'some patients are afraid of other patients'). Results also highlighted that some residents required support to understand the Likert scale response format. These difficulties often resulted in clinicians providing further explanations or using pictorial aids. This can create problems as clinicians may interpret and explain the questions in different ways or use different pictorial aids, potentially changing the meaning of the questions and resulting in reduced validity and reliability of the questionnaire.

In 2012 Alderman and Groucott examined whether the EssenCES is suitable for use in a UK neurobehavioural rehabilitation setting, including 76 staff and 38 residents. Although not conducted in IDD settings, there are a number of commonalities in the cognitive difficulties experienced by individuals with IDD and those affected by acquired brain injury (ABI). For example, both individuals with $\mathrm{ABI}$ and IDD often experience difficulties with comprehension and expression of language, short-term memory problems and reduced information processing abilities (Healthcare Improvement Scotland, 2013; Royal College of Physicians and British Society of Rehabilitation Medicine, 2003). Findings were similar to those obtained by Howells et al. (2009) and Tonkin et al. (2012) in terms of supporting the existing factor structure of the EssenCES and demonstrating satisfactory internal consistency. Convergent validity was also demonstrated through statistically significant relationships between the EssenCES and other measures of social climate. However, the authors also undertook a Rasch Analysis ${ }^{3}$ in order to test the assumption that the EssenCES can be classed as true interval-scale measurement tool. Their findings highlighted erratic and unpredictable

\footnotetext{
${ }^{3}$ Rasch Analysis is a unique approach of mathematical modeling based upon a latent trait and accomplishes stochastic (probabilistic) conjoint additivity (conjoint means measurement of persons and items on the same scale and additivity is the equal-interval property of the scale).
} 
response patterns along with category redundancy and disordered thresholds (suggesting that the subscales are not functioning as intended) across all items, mainly within the resident responses. They concluded that the EssenCES is unlikely to be a reliable and valid measure in neurobehavioural settings, with comments echoing those of Chester et al. (2015) including that some items are too complicated for those with cognitive impairments to understand, and that there are difficulties with the use of a five-point Likert scale.

The brief review above demonstrates the paucity of research examining social climate amongst IDD populations. In the wake of the Winterbourne View scandal and in line with relevant government strategies (e.g. Department of Health, 2001, 2009), it is vital that this lack of research be addressed to ensure that individuals with IDD are provided with the opportunity to express their opinions regarding the social climate of their units in a more independent manner, ultimately enhancing inclusion. The achievement of this aim, however, rests on reliable and valid questionnaires to measure the social climate in IDD settings. As demonstrated by Chester et al. (2015) and Alderman and Groucott (2012), there are significant questions regarding the suitability of existing questionnaire measures of social climate for this purpose. The next part of the review will consider in more detail the particular cognitive deficits experienced by IDD populations and the potential impact this might have on their ability to reliably complete questionnaire measures of social climate.

\section{IDD and self-reporting}

Individuals with IDD have significant impairments in intelligence (IQ <70) and deficits in social and adaptive functioning that present before adulthood (18 years) and have a lasting effect on development (American Psychiatric Association, 2013). There are a number of important core cognitive deficits associated with IDD which need to be considered when examining their ability to complete self-report questionnaire measures. However, it is also 
important to consider the strengths and abilities of individuals with IDD when considering how self-report measures can be adapted to improve their suitability for this population (Oliver, 1981).

Many of the widely reported difficulties in relation to completion of self-report measures in IDD are highly relevant to measures of social climate. Individuals are often limited in their cognitive processing ability (affecting short-term memory and information recall, and ability to organise, compare and contrast information), comprehension (impacting the ability to understand the meaning of questions, and the ability to grasp unfamiliar concepts and interpret complex sentences) and expression (ability to articulate a response to a question) (Emerson, Felce, \& Stancliffe, 2013). Individuals can present with difficulties understanding questions that include abstract, subjective or generalised concepts, or that are phrased in the negative or passive tense (Emerson et al., 2013; Finlay \& Lyons, 2001). Response biases and difficulties understanding response formats are common, with multipoint Likert scales being cited as more of a challenge for individuals with IDD due to, firstly, requiring individuals to hold multiple response options in their mind which, due to working memory difficulties, is more of a challenge for individuals with IDD and, secondly, to differentiate subtle differences between response options (Emerson et al., 2013; Fang et al, 2011; Kells, 2011).

\section{Cognitive difficulties}

Individuals with IDD can exhibit difficulties sustaining attention, with this becoming more prevalent when tasks place increased demands on encoding and memory abilities (Tomporowski \& Hager, 1992). The measures of social climate discussed above are of varying lengths, with the shortest being the EssenCES (17 items) and the longest the WAS/CIES (100/90 items respectively). Clearly, lengthy measures will present more 
challenges for individuals with IDD due to difficulties maintaining attention, particularly if these measures are more complex or if the individuals do not perceive the questionnaire to be of interest.

Memory deficits can be apparent in individuals with IDD, with difficulties retaining information in short-term memory and accessing information stored in long-term memory (Kells, 2011). Measures of social climate require individuals to retain the question and the response choices in their short-term memory, to organise and recall this information in order to provide a response. They also rely on individuals' ability to recall experiences and situations that have occurred previously. It is reported that memory difficulties are more prevalent when lengthy questions are asked (Kabzems, 1985) and, as measures of social climate include a number of questions which could be considered lengthy (e.g. 'If an inmate lets other people know that he does not want to be bothered, they will not bother him' (PEI)), it is likely that memory deficits may impact upon responses. Furthermore, although Tonkin and Howells (2011) state that questionnaire measures of social climate can be used for both longitudinal and cross-sectional monitoring, none of the measures discussed above stipulate whether the questionnaire should be completed in relation to a particular time period (e.g. the previous week, month or longer). Clearly this will be dependent on the reason for administering the measure. However, of note is that a lack of explicit guidelines may result in individuals with IDD struggling to understand exactly what time period is being referred to and which previous experiences and situations are in fact relevant to the questions.

\section{Linguistic difficulties}

Measures of social climate phrase their questions in different tenses and use differing personal pronouns. With regards to tenses, the PGCI and EssenCES use the present tense, the PEI uses a combination of present and future tense, and the CIES uses only the future tense. 
It is recommended that the present tense is used where possible when communicating with individuals with IDD (Gentile \& Gillig, 2012), thus individuals with IDD may have more difficulty responding to questions on the CIES and parts of the PEI than the PGCI or EssenCES. When communicating with individuals with IDD it is recommended that first person pronouns are used (Mencap, 2002). The PEI, CIES and EssenCES are written in the third person and use terms such as 'inmates', 'patients' and 'residents', whereas the PGCI is written in the first person, using 'I', 'me' and 'we'. This may reflect the fact that some measures are designed to be completed by both residents and staff; however, this can result in difficulties for individuals with IDD as, for example, they could interpret the term 'inmate' as meaning themselves, themselves and other inmates, or only other inmates, which can impact upon the meaning of the questions. Furthermore, some measures of social climate include contractions, for example, the PGCI includes the word 'don't' and the CIES includes both 'don't' and 'won't'. Using contractions rather than the full words (e.g. 'do not' or 'will not') can pose difficulties for some individuals with IDD as they may rely on the presence of the word 'not' to understand what is being said (Change, 2009).

Measures of social climate contain a number of questions which could be considered complex, for example 'Some patients are so excitable that one deals very cautiously with them' (EssenCES) and 'There will be very little emphasis on making residents more practical' (CIES). The use of complex questions poses difficulties for many individuals with ID (Kabzems, 1985) and can result in individuals with IDD responding based on single words contained in the question rather than to the question as a whole (Zetlin, Heriot, \& Turner, 1985). Social climate measures use questions that are phrased in the negative tense, for example 'Often, staff seem not to care if patients succeed or fail in treatment' (EssenCES), 'There will be very little emphasis on making plans for getting out of here' (CIES) and ‘Group workers don’t have enough time for me’ (PGCI). Individuals can find negatively 
phrased questions more difficult to respond to as they are constructed in a more complicated way and often include modifiers (single words or clauses that change the meaning of the questions). Individuals with IDD will at times ignore the modifier and answer the question as if it is phrased in the positive (Finlay \& Lyons, 2001). It has also been noted that individuals with IDD may be less likely to provide criticism, preferring to respond to more positive statements (Lowe \& de Paiva, 1988). Measures of social climate also contain questions whereby individuals are asked to comment on the views of others, for example 'At times, members of staff are afraid of some of the patients' (EssenCES) and 'The staff will know what the residents want' (CIES). This type of question can pose problems for individuals with IDD due to a limited ability to reflect on and understand the views and experiences of others (Jolliffe \& Farrington, 2004). Diagnoses such as Autistic Spectrum Disorder, present in approximately $30-40 \%$ of patients in forensic intellectual disability services (Esan, Chester, Gunaratna, Hoare, \& Alexander, 2015), can further exacerbate these difficulties, due to associated difficulties in theory of mind.

Some of the language used in existing social climate measures could be viewed as unsuitable for individuals with IDD due to the use of abstract concepts and generalised terms. Questions such as 'This ward has a homely atmosphere' (EssenCES), 'There will be very little group spirit on the unit' (CIES) and 'Group workers allow me some space' (PGCI) include words of an abstract nature and could be misunderstood by individuals with IDD. The majority of the questions on measures of social climate also require individuals with IDD to indicate what 'usually' happens on the unit or how they feel about situations on a 'general' basis. These types of questions can be difficult for individuals with IDD as they can struggle to aggregate specific instances and to use this information to form a general evaluation (Finlay \& Lyons, 2001). 
It is reported that individuals with IDD can have difficulty in making direct comparisons (Heal \& Sigelman, 1995). Some measures of social climate contain questions that require respondents to make these sorts of comparisons, for example 'Staff will care more about how residents feel than about their practical problems’ (CIES). Questions that include more than one clause and, therefore, more than one question can also be more difficult for individuals with IDD to understand (Beail \& Jahoda, 2012). Questions such as: 'Staff know patients and their personal histories very well' (EssenCES) and 'Group workers pay attention to me and respect my feelings' (PGCI) may therefore be more of a challenge for individuals with IDD.

\section{Response biases, response format difficulties and questionnaire administration}

There is a multitude of research that has examined response biases when using questionnaire measures in IDD populations, with authors reporting that response biases such as acquiescence (the tendency to say yes to questions regardless of content), recency bias (the tendency to select the last option mentioned in multiple-choice questions, irrespective of one's true opinion), nay-saying (saying no to every question) and suggestibility are more prevalent in IDD populations (Emerson et al. 2013; Kells, 2011). These response biases are more prevalent when individuals do not know the answer to the question that is posed and, are therefore more likely to occur when complex questions are asked (Kells, 2011). Clearly these response biases can reduce the reliability and validity of questionnaire measures in individuals with IDD (Kells, 2011).

The response formats used for social climate measures vary. The PGCI and the EssenCES use five-point Likert scales, the PEI a four-point Likert scale, and the CIES a forced-choice scale (true/false). The use of a four or five-point Likert scale is likely to compound the difficulties mentioned above as, although some studies have found that the 
number of response options does not affect response rate (Hartley \& Maclean, 2006), individuals with IDD have repeatedly been shown to struggle to respond effectively to four and five-point Likert scales, with evidence suggesting that a three-point scale is more appropriate (Fang et al., 2011; Sentell \& Ratcliff-Baird, 2003). Issues have also been identified when using forced-choice scales in IDD populations, with evidence suggesting that there is an increased likelihood of acquiescence when these scales do not include a response option of 'I don’t know' (Finlay \& Lyons, 2002). Of note is that the CIES does not offer such an option.

It is important to note that individuals with IDD are more likely to have the questionnaire presented orally rather than in a written format (Finlay \& Lyons, 2001). This poses a number of difficulties. Firstly, oral presentation of questionnaires with multiple response choices places pressure on short term memory (Kabzems, 1985). Secondly, the resulting reduction in anonymity of responses can increase the likelihood of nay-saying or socially desirable responding when individuals are asked questions regarding prohibited behaviour or the quality of their care (Finlay \& Lyons, 2001). Lastly, it has been suggested that oral presentation of questionnaire measures can lead to administrating staff providing further explanation of the questions, resulting in concerns surrounding staff projecting their own interpretation of a question on to individuals (Chester et al., 2015).

\section{Summary}

As a result of the difficulties detailed above, it is clear that many individuals with IDD will struggle when completing questionnaire measures of social climate. Although these difficulties will vary between level of IDD, different measures, questions and methods of administration, they can be expected to impact on the reliability and validity of such measures. It is therefore necessary to question the results of any measures of social climate 
with IDD populations as the findings may not accurately represent this populations' views. Self-report measures can be adapted to better suit IDD populations, and the next section of the paper will review these methods.

\section{Adapting measures of social climate for people with IDD}

Given the heterogeneity in cognitive ability seen within the IDD population (Finlay \& Lyons, 2001), it is clearly unrealistic to expect that self-report measures of social climate can be adapted in a way that would make them accessible to all individuals with IDD. A more realistic approach is to adapt self-report measures such that they can be completed by the majority of individuals with IDD. This method has been successfully achieved for a variety of measures with studies showing that the adaptations have resulted in reliable and valid measurement tools (e.g. the Social Intimacy Scale; Keeling, Rose, \& Beech, 2007; The Glasgow Anxiety Scale; Mindham \& Espie, 2003; The Modified Worker Loneliness Questionnaire; Stancliffe, Wilson, Bigby, Balandin, \& Craig, 2014). Results of the aforementioned studies show that a large number of those with IDD can reliably complete self-report measures regarding subjectiye states, suggesting that it is not unrealistic to expect that social climate measures can be successfully adapted for individuals with IDD. Whilst this approach may result in the exclusion of some, it appears this is the most inclusive way of gathering self-report data from IDD populations at present. Moreover, such an approach is clearly preferable to making no changes at all (Emerson et al., 2013).

Questionnaire measures can be adapted for IDD populations. A summary of the difficulties, along with possible adaptations derived from the literature are presented in Table 1. 
Table 1.

Summary of Difficulties and Possible Adaptations

Area of Difficulty

Linguistic content

Attention and memory

Response biases and response format

Administration
Possible Adaptation

Use the present tense.

Do not use contractions.

Ensure that all questions are written in the first person.

Simplify complex language.

Remove negatively phrased questions and questions that require respondents to make direct comparisons.

Replace abstract concepts with more concrete concepts where possible.

Do not use questions containing modifiers or more than one clause.

Replace generalised questions with questions that relate to a specific

situation or time-point.

ment on the views of

Remove questions that require respondents to comment on the views of

others.

Simplify the question content.

Shorten the questionnaire

Shorten the length of questions

Inform respondents of the time-frame that the questions relate to and

identify anchor events.

Use three-point Likert scales instead of four or five-point Likert scales.

Include pre-administration screening when using Likert-scales.

Include pictorial representations of the response choices when using both

Likert-scales and forced-choice scales.

Include a response option of 'I don't know' when using forced-choice scales.

$$
3 \text { ofipted phrasing of }
$$

Use scripted phrasing of questions and include alternatively worded scripted phrases.

Consider the layout of the questionnaire and the type and size of font used.
Reference

Gentile \& Gillig (2012)

Change (2009)

Mencap (2002)

Kabzems (1985); Zetlin, Heriot, \& Turner (1985)

Finlay \& Lyons (2001); Heal \& Sigelman (1995)

Finlay \& Lyons (2001)

Beail \& Jahoda (2012); Finlay \& Lyons (2001)

Finlay \& Lyons (2001)

Jolliffe \& Farrington (2004)

Finlay \& Lyons (2001)

Tomporowski \& Hager (1992)

Kabzems (1985); Prosser \& Bromley (2012)

Prosser \& Bromley (2012)

Fang et al. (2011); Sentell \& Ratcliff-Baird (2003)

Cummins, McCabe, Romeo, Reid, \& Waters (1997)

Hartley \& Maclean (2006)

Finlay \& Lyons (2002)

Finlay \& Lyons (2001); Hartley \& Maclean (2006)

Mencap (2002) 
Ensuring that measures are succinct will help to improve attention and increase motivation amongst individuals with IDD. This is particularly relevant to the CIES (90 items), PEI (48 items) and PGCI (36 items). With regards to the grammatical content of social climate measures, potential adaptations include posing all questions in the present tense, for example, replacing 'The residents will be proud of the unit' (CIES) with 'The residents are proud of the unit'. It is also important to ensure that questions do not include contractions. Lastly, it would be beneficial to ensure that all questions are written in the first person, replacing terms such as 'patients' and 'inmates' with terms such as ' $\mathrm{I}$ ' and 'we'. Although this would aid understanding of individuals with IDD, this would, however, impact on the ability of both residents and staff to complete these measures and may also alter the meaning of some questions, as clearly residents would relate the questions only to themselves and not the resident population as a whole. This is therefore something that would require careful consideration.

When considering the language used in measures of social climate, adaptations suggested in previous research (see, for example, Finlay \& Lyons, 2001) such as simplifying language, and removal of negatively phrased questions and questions requiring direct comparisons to be made could be employed. Abstract concepts could be replaced with more concrete concepts (where possible) and question structures that include modifiers or more than one clause could be reworded. Additionally, questions that require respondents to indicate what 'usually' happens on the unit or how they feel on a 'general' basis could be amended to ensure that they are structured to relate to specific situations or to respondents' feeling at a defined time point. Furthermore, if possible, it would be helpful to remove any questions that require respondents to comment on the views of others.

Simplifying questions in this manner will improve respondents' understanding of the questions; reducing the short-term memory load required of respondents and, therefore, 
increasing the likelihood that participant responses will be reliable and valid. With regards to the presence of long-term memory deficits in individuals with IDD, it would be of benefit for those administering measures of social climate to inform individuals with IDD of the timeframes of which the questions relate to and to identify anchor events (events that have occurred in the recent past that can be remembered accurately), as this can be used to aid individuals to understand the relevant time-frame (Prosser \& Bromley, 2012).

The response format for an IDD-specific measure of social climate would also need to be adapted. The four-point and five-point Likert scales used in the PGCI,PEI and EssenCES could be replaced with three-point Likert scales, as this has been shown to be more appropriate for IDD populations (Fang et al., 2011; Sentell \& Ratcliff-Baird, 2003), although of note is that this adaptation would alter the scoring, and would therefore impact on the ability to compare scores from the original and the adapted versions. Pre-administration screening could also be incorporated into the PGCI, PEI and EssenCES in order to determine which individuals are competent at distinguishing between the response options and are therefore more likely to provide reliable and valid responses. When using Likert scales, it has been shown that the use of pre-administration screening results in improved reliability and validity of responses in IDD populations (Cummins, McCabe, Romeo, Reid, \& Waters, 1997, Hartley \& Maclean, 2006). Naturally, this could result in individuals with more moderate IDD being excluded, but this would help to ensure that reliable and valid insights into resident experience and the social climate are gained (Emerson et al., 2013). Pictorial representations of Likert scale response choices could also be included, as it is reported that this can support individuals with IDD to better distinguish between subtle differences among response choices (Hartley \& Maclean, 2006), for example, between 'not at all' and 'little' and between 'quite a lot' and 'very much' (EssenCES). Evidence suggests that this can also result in an increased response rate among individuals with IDD (Heal \& Sigelman, 1985; 
Sigelman, Budd, Winer, Schoenrock, \& Martin, 1982). The forced choice response scale used in the CIES could also include pictorial representations of response choices, as this has been shown to increase response rate and to reduce recency bias (Heal \& Sigelman, 1985) Additionally, inclusion of an additional response option of 'I don’t know' alongside the existing true and false options of the CIES could reduce acquiescence (Finlay \& Lyons, 2002).

Whilst the above suggestions are likely to improve the suitability of social climate measures for individuals with IDD, it is also necessary to consider how these measures should be administered in order to ensure appropriate communication support is being provided to individuals with IDD. From July 2016 onwards it is a legal requirement for all UK organisations that provide NHS or publicly funded adult social care to conform to the Accessible Information Standards (NHS Accessible Information Specification; 2015). Although this remains a relatively under-researched area, meaning suggestions regarding the most appropriate way of developing IDD-specific administration guidelines are few, some authors such as Finlay and Lyons (2001) and Hartley and Maclean (2006) have highlighted ways of improving questionnaire administration in IDD populations which are of relevance to measures of social climate. The use of scripted phrasing of questions and of providing alternative scripted phrases to enable questions to be explained in a different way have been discussed in the literature (Finlay \& Lyons, 2001; Hartley \& Maclean, 2006). If used within measures of social climate, this would provide an additional way of checking respondents' understanding whilst preventing administrators from projecting their interpretation of the questions on to respondents. Furthermore, in terms of ensuring provision of accessible information, it would be important for questionnaire developers to consider details such as the layout of the questionnaire and the type and size of font used (Mencap, 2002). 
It is also necessary to note that, at present, it is not clear which aspects of social climate are important to individuals with IDD. Thus, the definitions of social climate included within current measures may not be relevant within forensic IDD services. It is therefore important that future research seek to empirically examine the factor structure of any adapted social climate questionnaires, rather than assuming that the same structure will exist across IDD and non-IDD populations.

\section{Future research}

Evidently, development of an IDD-specific measure of social climate woutd help to fill a gap in the forensic social climate literature. The authors have recently commenced an international, multi-site research project looking at adapting the EssenCES for use with individuals with IDD. The project is comprised of two phases. Phase one involves using questionnaires, interviews and focus groups to identify issues encountered by staff when utilising the EssenCES within forensic IDD settings. This will identify aspects of the EssenCES that need changing and how the questionnaire might be adapted to make it more suitable for use with IDD populations. The second phase involves developing an IDDspecific version of the EssenCES. This new questionnaire will be piloted with staff and residents before a final version is produced and trialled within a large number of forensic LD services. Psychometric validity and the relationship between social climate and important clinical and organisational outcomes will also be examined.

There are clearly a wide range of benefits associated with developing this IDD-specific measure of social climate. Firstly, this measure would have a valuable contribution towards empowering those with IDD. In line with the key principles highlighted by the Department of Health (2001), an accessible measure of social climate would promote the rights of those with IDD to express their opinions and preferences in a more independent manner, ultimately 
enhancing inclusion. Clinically, the use of a measure that demonstrates reliability and validity in IDD populations would improve understanding of the social climate construct within IDD settings, extending knowledge of the aspects of social climate important to those with IDD and facilitating examination of the relationships between social climate and other clinical and organisational outcomes. This would contribute positively to service evaluation, highlighting ideas for service development that would ultimately help to improve the rehabilitation of offenders with IDD. 


\section{References}

Alderman, N., \& Groucott, L. (2012). Measurement of social climate within neurobehavioural rehabilitation services using the EssenCES. Neuropsychological Rehabilitation, 22, 768-93. doi: 10.1080/09602011.2012.689683

American Psychiatric Association. (2013). Diagnostic and statistical manual of mental disorders $\left(5^{\text {th }}\right.$ ed.). Arlington, VA: American Psychiatric Association.

Beail, N., \& Jahoda, A. (2012). Working with people: Direct interventions. In E. Emerson, C. Hatton, K. Dickson, R. Gone, A. Caine and J. Bromley (Eds.), Clinical psychology and people with intellectual disabilities (pp. 121-139). Chichester, UK: John Wiley \& Sons, Ltd.

Beech, A.R., \& Hamilton-Giachritsis, C. (2005). Relationship between therapeutic climate and treatment outcome in group-based sexual offender treatment programs. Sexual Abuse: A Journal of Research and Treatment, 17, 127-140. doi:

$10.1177 / 107906320501700204$

Bradford, A. R. (2006). An Examination of the prison environment: An analysis of inmate concerns across eight environmental dimensions (Unpublished doctoral dissertation). East Tennessee State University, USA.

Bressington, D., Stewart, B., Beer, D., \& MacInnes, D. (2011). Levels of service user satisfaction in secure settings - A survey of the association between perceived social climate, perceived therapeutic relationship and satisfaction with forensic services. International Journal of Nursing Studies, 48, 1349-1356. doi:

10.1016/j.ijnurstu.2011.05.011 
Change. (2009). How to make information accessible: A guide to producing easy read documents. Retrieved from http://www.accessibleinfo.co.uk/information/moreinformation.php

Chester, V., McCathie, J., Quinn, M., Ryan, L., Popple, J., Loveridge, C., \& Spall, J. (2015). Clinician experiences of administering the Essen Climate Evaluation Schema (EssenCES) in a forensic intellectual disability service. Advances in Mental Health and Intellectual Disabilities, 9, 70-78. doi: 10.1108/AMHID-06-2014-0024

Cummins, R.A., McCabe, M.P., Romeo, Y., Reid, S., \& Waters, L. (1997). An initial evaluation of the Comprehensive Quality of Life Scale-Intellectual Disability. International Journal of Disability, Development and Education, 44, 7-19. doi: $10.1080 / 0156655970440102$

Day, A., Casey, S., Vess, J., \& Huisy, G. (2012). Assessing the therapeutic climate of prisons. Criminal Justice and Behaviour, 39, 156-168. doi: 10.1177/0004865813518543

Department of Health. (2001). Valuing people: A new strategy for learning disability for the 21st century. Retrieved from http://webarchive.nationalarchives.gov.uk/20130107105354/http:/www.dh.gov.uk/pro d_consum_dh/groups/dh_digitalassets/documents/digitalasset/dh_093375.pdf

Department of Health. (2009). Valuing people now: a new three-year strategy for people with learning disabilities. Retrieved from http://webarchive.nationalarchives.gov.uk/20130107105354/http:/www.dh.gov.uk/pro d_consum_dh/groups/dh_digitalassets/documents/digitalasset/dh_093375.pdf

Emerson, E., Felce, D., \& Stancliffe, R.J. (2013). Issues concerning self-report data and population-based datasets involving people with intellectual disabilities. Intellectual and Developmental Disabilities, 51, 333-348. doi: 10.1352/1934-9556-51.5.333 
Esan, F., Chester, V., Alexander, R. T., Gunaratna, I. J., \& Hoare, S. (2015). An analysis of the clinical, forensic, and treatment outcome factors of those with autistic spectrum disorders treated in forensic intellectual disability settings. Journal of Applied Research in Intellectual Disabilities, 28, 193-200. doi: 10.1111/jar.12121

Fang, J., Fleck, M.P., Green, A., McVilly, K., Hao, Y., Tan, W., ... Power, M. (2011). The response scale for the intellectual disability module of the WHOQOL: 5-point or 3point? Journal of Intellectual Disability Research, 55, 537-549. doi: 10.1111/j.13652788.2011.01401.X

Finlay, W.M.L., \& Lyons, E. (2001). Methodological challenges in interviewing and using self-report questionnaires with people with mental retardation. Psychological Assessment, 13, 319-335. doi: 10.1037/1040-3590.13.3.319

Finlay, W.M.L, \& Lyons, E. (2002). Acquiescence in interviews with people who have mental retardation. Mental Retardation, 40, 14-29. doi: 10.1352/0047-6765(2002)040

Friis, S., \& Helldin, L. (1994). The contribution made by the clinical setting to violence among psychiatric patients. Criminal Behaviour and Mental Health, 4, 341-352. Retrieved from http://onlinelibrary.wiley.com .ezproxy.lib.le.ac.uk/journal/10.1002/(ISSN)1471-2857/issues

Gentile, J.P., \& Gillig, M.P. (2012). Interviewing techniques. In J.P. Gentile \& P.M. Gillig (Eds.), Psychiatry of intellectual disability: A Practical Manual (pp. 90-124). Chichester, UK: John Wiley \& Sons, Ltd.

Hartley, S.L., \& Maclean Jr, W.E. (2006). A review of the reliability and validity of likerttype scales for people with intellectual disability. Journal of Intellectual Disability Research, 50, 813-827. doi: 10.1111/j.1365-2788.2006.00844.x 
Heal, L.W., \& Sigelman, C.K. (1995). Response biases in interviews of individuals with limited mental ability. Journal of Intellectual Disability Research, 39, 331-340. doi: 10.1111/j.1365-2788.1995.tb00525.x

Health and Social Care Information Centre. (2015). Learning disability census report. Retrieved from http://www.hscic.gov.uk/catalogue/PUB19428/ld-census-initialsep15-rep.pdf

Healthcare Improvement Scotland. (2013). Brain injury rehabilitation in adults (Sign 130). Retrieved from http://www.sign.ac.uk/pdf/sign130.pdf

Heynen, E.J.E. (2016). No guts, no gains! The relation between living group climate and social development of juvenile delinquents in detention (Unpublished doctoral dissertation). The University of Amsterdam, Amsterdam.

Howells, K., Tonkin, M., Milburn, C., Lewis, J., Draycott, S., Cordwell, J., ... Schalast, N. (2009). The EssenCES measure of social climate: A preliminary validation and normative data in UK high secure hospital settings. Criminal Behaviour and Mental Health, 19, 308-320. doi: 10.1002/cbm.745

Jolliffe, D., \& Farrington, D.P. (2004). Empathy and offending: A systematic review and meta-analysis. Aggression and Violent Behaviour, 9, 441-476. doi: 10.1016/j.avb.2003.03.001

Kabzems, V. (1985). The use of self-report measures with mentally retarded individuals. Mental Retardation and Learning Disability Bulletin, 13, 106-114. Retrieved from: http://psycnet.apa.org/psycinfo/1986-20543-001 
Keeling, J.A., Rose, J.L., \& Beech, A.R. (2007). A preliminary evaluation of the adaptation of four assessments for offenders with special needs. Journal of Intellectual and Developmental Disability, 32, 62-73. doi: 10.1080/13668250701378538

Kells, M. (2011). The psychometric assessment of offenders with an intellectual disability (Doctoral dissertation). Retrieved from EThOS database (uk.bl.ethos.542109)

Langdon, P.E., Swift, A., \& Budd, R. (2006). Social climate within secure inpatient services for people with intellectual disabilities. Journal of Intellectual Disability Research, 50, 828-836. doi: 10.1111/j.1365-2788.2006.00847.x

Long, C.G., Anagnostakis, K., Fox, E., Silaule, P., Somers, J., West, R., \& Webster, A. (2011). Social climate along the pathway of care in women's secure mental health service: Variation with level of security, patient motivation, therapeutic alliance and level of disturbance. Criminal Behaviour and Mental Health, 21, 202-214. doi: 10.1002/cbm.791

Lowe, K., \& de Paiva, S. (1988). Canvassing the views of people with a mental handicap. Irish Journal of Psychology, 9, 220-234. doi: 10.1080/03033910.1988.10557718

McGee, M.G., \& Woods, D.J. (1978). Use of Moos' Ward Atmosphere Scale in a residential setting for mentally retarded adolescents. Psychological Reports, 43, 580-582. doi: 10.2466/pr0.1978.43.2.580

Mencap. (2002). Am I making myself clear? Mencap's guidelines for accessible writing. Retrieved from https:/www.mencap.org.uk/sites/default/files/documents/200907/Am\%20\%20I\%20\%20making\%20\%20myself\%20\%20clear\%20[1].pdf

Milsom, S.A., Freestone, M., Duller, R., Bouman, M., \& Taylor, C. (2014). Factor structure of the Essen Climate Evaluation Schema (EssenCES) measure of social climate in a 
UK medium security setting. Criminal Behaviour and Mental Health, 24, 86-99. doi: 10.1002/cbm.1878

Mindham, J., \& Espie, C.A. (2003). Glasgow Anxiety Scale for people with an intellectual disability (GAS-ID): Development and psychometric properties of a new measure for use with people with mild intellectual disability. Journal of Intellectual Disability Research, 47, 22-30. doi: 10.1046/j.1365-2788.2003.00457.x

Moos, R.H. (1974). Ward Atmosphere Scale Manual. Palo Alto, CA: Consulting Psychologists Press.

Moos, R.H. (1975). Evaluating Correctional and Community Settings. New York: John Wiley and Sons.

Moos, R.H. (1987). Correctional Institutions Environment Scale. Palo Alto, CA: Consulting Psychologists Press.

Moos, R.H. (1987b). The Social Climate Scales: A User's Guide. Palo Alto, CA: Consulting Psychologists Press.

Moos, R.H., \& Houts, P.S. (1968). Assessment of the social atmospheres of psychiatric wards. Journal of Abnormal Psychology, 73, 595-604. doi: 10.1037/h0026600

Moos, R.H., \& Schaefer, J.A. (1987). Evaluating health care work settings: A holistic conceptual framework. Psychology \& Health, 1, 97-122. doi: $10.1080 / 08870448708400318$

Moos, R.H., Shelton, R., \& Petty, C. (1973). Perceived ward climate and treatment outcome. Journal of Abnormal Psychology, 82, 291-298. doi: 10.1037/h0035184

Mottram, P. G. (2007). HMP Liverpool, Styal and Hindley Study Report. Liverpool: University of Liverpool. 
National Health Service (NHS) England. (2015). Accessible information specification.

Retrieved from https://www.england.nhs.uk/wp-content/uploads/2015/07/accessinfo-spec-fin.pdf

Oliver, M., J. (1981). A new perspective for social workers. In J. Campling (Eds.), The handicapped person in the community (pp. 25-59). London: Radar.

Prison Reform Trust. (2007). Prisoners' voices: Experiences of the criminal justice system by prisoners with learning disabilities and difficulties. Retrieved from http://www.prisonreformtrust.org.uk/Portals/0/Documents/No\%20One\%20Knows\%2 0report-2.pdf

Prosser, H., \& Bromley, J. (2012). Interviewing people with intellectual disabilities. In E. Emerson, C. Hatton, K. Dickson, R. Gone, A. Caine and J. Bromley (Eds.), Clinical psychology and people with intellectual disabilities (pp. 121-139). Chichester, UK: John Wiley \& Sons, Ltd.

Quinn, M., Thomas, C., \& Chester, V. (2012). The Essen Climate Evaluation Schema measure of social climate in a secure service for people with intellectual disabilities. Advances in Mental Health and Intellectual Disabilities, 6, 171-178. doi: $10.1108 / 20441281211236562$

Rack, J. (2005). The Incidence of Hidden Disabilities in the Prison Population. Egham, Surrey: Dyslexia Institute.

Rose, J. (1993). Stress and staff in residential settings: the move from hospital to the community. Journal of Applied Research in Intellectual Disabilities, 6, 312-332. doi: 10.1111/j.1468-3148.1993.tb00062.x 
Røssberg, J.I., \& Friis, S. (2004). Patients' and staff's perceptions of the psychiatric ward environment. Psychiatric Services, 55, 798-803. doi: 10.1176/appi.ps.55.7.798

Royal College of Psychiatrists. (2013). People with learning disability and mental health, behavioural or forensic problems: the role of in-patient services. Retrieved from http://www.rcpsych.ac.uk/pdf/FR\%20ID\%2003\%20for\%20website.pdf

Royal College of Physicians and British Society of Rehabilitation Medicine. (2003).

Rehabilitation following acquired brain injury: National clinical guidelines. London:

RCP, BSRM.

Schalast, N., Redies, M., Collins, M., Stacey, J., \& Howells, K. (2008). EssenCES, a short questionnaire for assessing the social climate of forensic psychiatric wards. Criminal Behaviour and Mental Health, 18, 29-58. doi: 10.1002/cbm.677

Sentell T. L., \& Ratcliff-Baird B. (2003), Literacy and comprehension of Beck Depression Inventory response alternatives. Community Mental Health Journal, 39, 323-331. doi: 10.1023/A:1024072108467

Sigelman, C.K., Budd, E.C., Winer, J.L., Schoenrock, C.J., \& Martin, P.W. (1982). Evaluating alternative techniques of questioning mentally retarded persons. American Journal of Mental Deficiency, 86, 511-518. Retrieved from http://psycnet.apa.org/psycinfo/1982-21433-001

Stancliffe, R.J., Wilson, N.J., Bigby, C., Balandin, S., \& Craig, D. (2014). Responsiveness to self-report questions about loneliness: a comparison of mainstream and intellectual disability-specific instruments. Journal of Intellectual Disability Research, 58, 399405. doi: 10.1111/jir.12024

Toch, H. (1977). Living in prison: The ecology of survival. New York: The Free Press. 
Tomporowski, P.D. \& Hager, L.D. (1992). Sustained attention in mentally retarded individuals. In N.W. Bray (Ed.), International review of research in mental retardation (pp. 111-136). San Diego: Academic Press.

Tonkin, M. (2015). A review of questionnaire measures for assessing the social climate in prisons and forensic psychiatric hospitals. International Journal of Offender Therapy and Comparative Criminology, 1-30. doi: 10.1177/0306624X15578834

Tonkin, M., \& Howells, K. (2011). Evaluating regimes and environments. In K. Sheldon, J. Davies, \& K. Howells (Eds.), Research in practice for forensic professionals (pp. 249-270). Abington, UK: Routledge.

Tonkin, M., Howells, K., Ferguson, E., Clark, A, Newberry, M., \& Schalast, N. (2012). Lost in translation? Psychometric properties and construct validity of the English Essen Climate Evaluation Schema (EssenCES) social climate questionnaire. Psychological Assessment, 24, 573-580. doi: 10.1037/a0026267

Van der Helm, P., Stams, G.J., \& van der Laan, P. (2011). Measuring group climate in prison. The Prison Journal, 91, 158-176. doi: 10.1177/0032885511403595

Van der Helm, P., Stams, G.J., van Genabeek, M., \& van der Laan, P. (2012). Group climate, personality, and self-reported aggression in incarcerated male youth. The Journal of Forensic Psychiatry and Psychology, 23, 23-29. doi: 10.1080/14789949.2011.633615

Willets, L., Mooney, P., \& Blagden, N. (2014) Social climate in learning disability services. Journal of Intellectual Disabilities and Offending Behaviour, 5, 24-37. doi: 10.1108/JIDOB-10-2013-0025

Wright, K. N. (1985). Developing the prison environment inventory. Journal of Research in Crime and Delinquency, 22, 257-277. doi: 10.1177/0022427885022003005 
Wright, K. N., \& Boudouris, J. (1982). An assessment of the Moos Correctional Institutions Environment Scale. Journal of Research in Crime and Delinquency, 19, 255-276. doi: $10.1177 / 002242788201900208$

Zetlin, A. G., Heriot, M. J., \& Turner, J. L. (1985). Self-concept measurement in retarded adults: A micro-analysis of responses. Applied Research in Mental Retardation, 6, 113-125. doi: 10.1016/S0270-3092(85)80065-5 